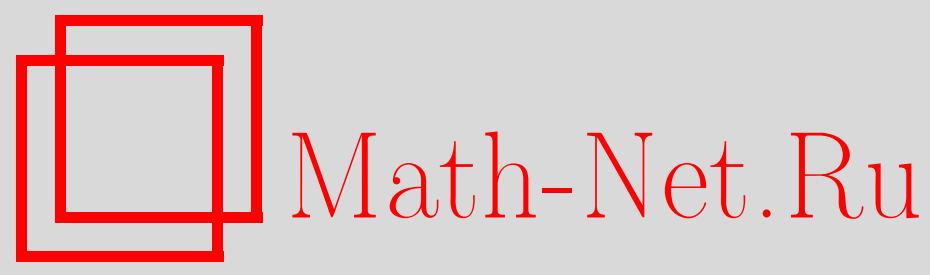

В. В. Зудилин, О целочисленности степенных разложений, связанных с гипергеометрическими рядами, Матем. заметки, 2002, том 71, выпуск 5, 662-676

DOI: https://doi.org/10.4213/mzm375

Использование Общероссийского математического портала Math-Net.Ru подразумевает, что вы прочитали и согласны с пользовательским соглашением http://www . mathnet.ru/rus/agreement

Параметры загрузки:

IP : 3.85 .5 .30

26 апреля 2023 г., 10:41:50

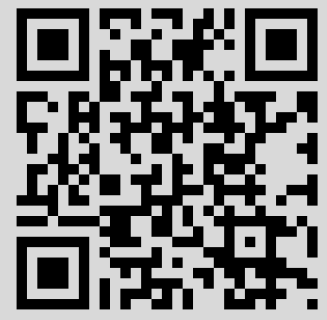




\section{О ЦЕЛОЧИСЛЕННОСТИ СТЕПЕННЫХ РАЗЛОЖЕНИЙ, СВЯЗАННЫХ С ГИПЕРГЕОМЕТРИЧЕСКИМИ РЯДАМИ}

В.В. Зудилин

В настоящей работе исследуются арифметические свойства степенных разложений, связанных с обобщенными гипергеометрическими дифференциальными уравнениями и рядами. Определяя ряды $f(z), g(z)$ по степеням $z$ таким образом, что $f(z)$ и $f(z) \log z+g(z)$ удовлетворяют гипергеометрическому уравнению при специальном выборе параметров, мы доказьваем, что ряд $q(z)=z e^{g(C z) / f(C z)}$ по степеням $z$ и его обрашение $z(q)$ по степеням $q$ имеют целочисленные коэффициенты (постоянная $C$ зависит от параметров гипергеометрического уравнения). Целочисленность разложения $z(q)$ для дифференциальных уравнений второго и третьего порядка является классическим результатом; для порядка выше 3 частичные результаты были недавно установлены Лианом и Яу. В своем доказательстве, пользуясь $p$-адической техникой Дворка, мы обобщаем схему их рассуждений.

Библиография: 11 названий.

1. Введение. Зафиксируем произвольное целое число $N \geqslant 2$, и пусть $N=p_{1}^{s_{1}} \times$ $p_{2}^{s_{2}} \cdots p_{l}^{s_{l}}$ - его разложение на простые множители, а целые $q_{1}, q_{2}, \ldots, q_{k}, 1=q_{1}<q_{2}<$ $\cdots<q_{k}<N$, образуют полньй набор остатков при делении на $N$, взаимно простых с самим числом $N$. Количество чисел $k$ в этом наборе задается хорошо известной формулой

$$
k=N\left(1-\frac{1}{p_{1}}\right)\left(1-\frac{1}{p_{2}}\right) \cdots\left(1-\frac{1}{p_{l}}\right)
$$

(см., например, [1, отд. 8, задача 25]). В заданных обозначениях справедливо следующее утверждение.

ЛЕмма 1. Для иелого $N \geqslant 2$ определим полохительную постоянную

$$
C_{N}:=N^{k} \cdot \prod_{p \mid N} p^{k /(p-1)}=\left(\prod_{j=1}^{l} p_{j}^{s_{j}+1 /\left(p_{j}-1\right)}\right)^{k},
$$

которая является чельм числом, поскольку $(p-1) \mid k$ для любого $p \mid N$ согласно (1). Тогда для любого иелого $m \geqslant 0$ число

$$
A(m)=A_{N}(m):=C_{N}^{m} \cdot \frac{\left(q_{1} / N\right)_{m}\left(q_{2} / N\right)_{m} \cdots\left(q_{k} / N\right)_{m}}{m !^{k}}
$$

Работа вьполнена при частичной поддержке объединенного проекта фонда INTAS и Российского фонда фундаментальных исследований, грант № IR-97-1904, и Государственной научной стипендии Российской АН для молодых ученых. 
является целым (положстельным) числом. Здесь $(x)_{m}=x(x+1) \cdots(x+m-1)$ для $m \geqslant 1 u(x)_{m}=1$ обозначает символ Похгаммера.

Мы доказываем это утверждение в п. 3, но для иллюстрации приведем два простых частных случая. Если $N=p^{s}$ является степенью простого и, значит, $k=p^{s}-p^{s-1}$, то выбор $C_{N}=p^{s p^{s}-(s-1) p^{s-1}}$ приводит к целым числам

$$
A(m)=\frac{\left(p^{s} m\right) !}{\left(p^{s-1} m\right) ! m ! p^{s}-p^{s-1}}, \quad m=0,1,2, \ldots
$$

Если $N=p_{1} p_{2}$, то $k=\left(p_{1}-1\right)\left(p_{2}-1\right)$ и выбор $C_{N}=p_{1}^{p_{1}\left(p_{2}-1\right)} p_{2}^{\left(p_{1}-1\right) p_{2}}$ дает целые числа

$$
A(m)=\frac{\left(p_{1} p_{2} m\right) !}{\left(p_{1} m\right) !\left(p_{2} m\right) ! m ! p_{1} p_{2}-p_{1}-p_{2}}, \quad m=0,1,2, \ldots
$$

Согласно лемме 1 обобщенный гипергеометрический ряд

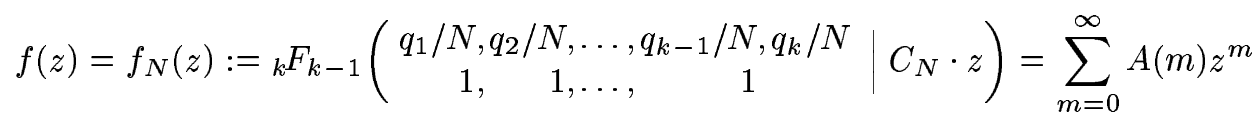

имеет целые коэффициенты в разложении по степеням $z$.

Полагая

$$
\begin{gathered}
D(x, m)=\frac{d}{d x} \log (x)_{m}=\sum_{n=1}^{m} \frac{1}{x+n-1}, \quad x \in(0,1], \quad m=0,1,2, \ldots, \\
D(m)=D_{N}(m):=\sum_{j=1}^{k} D\left(\frac{q_{j}}{N}, m\right)-k D(1, m), \quad m=0,1,2, \ldots,
\end{gathered}
$$

рассмотрим также степенной ряд

$$
g(z)=g_{N}(z):=\sum_{m=0}^{\infty} A(m) D(m) z^{m}=\sum_{m=1}^{\infty} A(m) D(m) z^{m}
$$

коэффициенты которого, вообще говоря, уже не являются цельми числами. Оба ряда $(5),(6)$ сходятся в окрестности точки $z=0$ (более точно, при $|z|<1 / C_{N}$ ); кроме того, при $N>2$ в этой окрестности функции $f(z)$ и $f(z) \log z+g(z)$ являются линейно независимыми решениями линейного однородного дифференциального уравнения

$$
\left(\left(z \frac{d}{d z}\right)^{k}-C_{N} \cdot z\left(z \frac{d}{d z}+\frac{q_{1}}{N}\right)\left(z \frac{d}{d z}+\frac{q_{2}}{N}\right) \cdots\left(z \frac{d}{d z}+\frac{q_{k}}{N}\right)\right) y=0 .
$$

Уравнение (7) является обобщенным гипергеометрическим уравнением, решения которого обладают максимальной унипотентной монодромией в смысле Моррисона (см. $[2, \S 1],[3, \S 4.2])$. 
Теорема 1. Пусть для заданного иелого $N \geqslant 2$ степенные ряды $f(z)=f_{N}(z)$, $g(z)=g_{N}(z)$ определяются формулами (3)-(6). Тогда коэффициенты степенного разложения

$$
q(z):=\exp \left(\frac{f(z) \log z+g(z)}{f(z)}\right)=z \cdot \exp \left(\frac{g(z)}{f(z)}\right)
$$

являются иелыми числами.

В случае простого $N$ данная теорема была доказана Лианом и Яу [4, теорема 5.5] с помощью $p$-адической техники Дворка [5]. В настоящей работе мы упрощаем метод работы [4] и доказьваем более общие результаты. Однако, по-настоящему общее утверждение, мотивированное результатами работы [5] и численными экспериментами, может быть сформулировано в следующем виде.

ГИПотЕЗА. Пусть $N_{1}, N_{2}, \ldots, N_{r}$ - иелье числа, $N_{j} \geqslant 2$ для всех $j=1, \ldots, r$; числовым последовательностям

$$
\begin{gathered}
A(m)=A_{N_{1}}(m) A_{N_{2}}(m) \cdots A_{N_{r}}(m), \quad m=0,1,2, \ldots, \\
D(m)=D_{N_{1}}(m)+D_{N_{2}}(m)+\cdots+D_{N_{r}}(m), \quad m=0,1,2, \ldots,
\end{gathered}
$$

отвечают степенные ряды

$$
f(z):=\sum_{m=0}^{\infty} A(m) z^{m}, \quad g(z):=\sum_{m=1}^{\infty} A(m) D(m) z^{m} .
$$

Тогда коэффиииенты степенного разложения

$$
q(z):=z \cdot \exp \left(\frac{g(z)}{f(z)}\right)
$$

являются иелыми числами.

СлЕДСТВИЕ. Для произвольного $N \geqslant 2$ определим степенные ряды

$$
\begin{aligned}
f(z) & :={ }_{N-1} F_{N-2}\left(\begin{array}{c}
1 / N, 2 / N, \ldots,(N-2) / N,(N-1) / N \\
1, \quad 1, \ldots,
\end{array} \mid N^{N} \cdot z\right)=\sum_{m=0}^{\infty} \frac{(N m) !}{m !^{N} z^{m},}(11) \\
g(z) & :=\sum_{m=1}^{\infty} \frac{(N m) !}{m !^{N}}\left(\sum_{j=1}^{N-1} D\left(\frac{j}{N}, m\right)-(N-1) D(1, m)\right) z^{m} .
\end{aligned}
$$

Тогда коэффициенты степенного разложсения

$$
q(z):=z \cdot \exp \left(\frac{g(z)}{f(z)}\right)
$$

являются целыми числами.

Для вьвода следствия достаточно в качестве набора целых чисел $\left\{N_{j}\right\}_{j=1, \ldots, r}$ в условиях гипотезы выбрать

$$
\left\{p_{1}^{\alpha_{1}} p_{2}^{\alpha_{2}} \cdots p_{l}^{\alpha_{l}}: 0 \leqslant \alpha_{j} \leqslant s_{j}, j=1, \ldots, l, \alpha_{1}+\alpha_{2}+\cdots+\alpha_{l}>0\right\},
$$

где $N=p_{1}^{s_{1}} p_{2}^{s_{2}} \cdots p_{l}^{s_{l}}$ - разложение числа $N$ на простые множители.

Наш вклад в частичное решение гипотезы может быть сформулирован следующим образом. 
Теорема 2. Пусть $N_{1}, N_{2}, \ldots, N_{r}$ - иелые числа, $N_{j} \geqslant 2$, при этом любое простое $p$, делящее произведение $N_{1} N_{2} \cdots N_{r}$, делит каждое $N_{j}, j=1, \ldots, r$ (например, $N_{1}=N_{2}=\cdots=N_{r}$ ). Определим степенные ряды $f(z), g(z)$ в соответствии с формулами (8)-(10). Тогда коэффичиенты степенного разложения

$$
q(z):=z \cdot \exp \left(\frac{g(z)}{f(z)}\right)
$$

являются чельми числами.

Из теоремы 2 мы получаем справедливость следствия из гипотезы для любого целого, являющегося степенью простого: $N=p^{s}$.

Теорема 3. Пусть $r \geqslant 1$ челое $u N=p^{s}$ для простого $p$ u иелого $s \geqslant 1$, cmeпенные ряды $f(z)$ и $g(z)$ определяются формулами

$$
\begin{aligned}
& f(z):=\sum_{m=0}^{\infty}\left(\frac{(N m) !}{m !^{N}}\right)^{r} z^{m} \\
& g(z):=r \sum_{m=1}^{\infty}\left(\frac{(N m) !}{m !^{N}}\right)^{r}\left(\sum_{j=1}^{N-1} D\left(\frac{j}{N}, m\right)-(N-1) D(1, m)\right) z^{m} .
\end{aligned}
$$

Тогда коэффичиенты степенного разложсения

$$
q(z):=z \cdot \exp \left(\frac{g(z)}{f(z)}\right)
$$

являются иельми числами.

ДокаЗАТЕльСтво. Выбирая $r$ копий множества $\left\{p^{\alpha}\right\}_{1 \leqslant \alpha \leqslant s}$ в качестве набора $\left\{N_{j}\right\}$ в теореме 2 , получаем требуемое утверждение.

Гипергеометрические ряды $f(z)$ из (4), (10)-(12) и линейные дифференциальные уравнения, которьм они удовлетворяют, естественным образом появляются в геометрии многообразий Калаби-Яу. Так, например, периоды семейства $(N-2)$-мерных гиперповерхностей

$$
\mathscr{Q}_{\psi}:=\left\{x_{1}^{N}+\cdots+x_{N}^{N}-N \psi x_{1} \cdots x_{N}=0\right\} \subset \mathbb{P}^{N-1}
$$

как функции от $z=(N \psi)^{-N}$ удовлетворяют тому же дифференциальному уравнению, что и ряд (11) (см., например, [6, следствие $(2.3 .8 .1)],[7, \S 8.3])$. Обращение $z(q)$ ряда $q(z)=z e^{g(z) / f(z)}$, представляющее аналитическую функцию в некоторой окрестности $q=0$, называется зеркальным отображением для соответствующего семейства гиперповерхностей.

Лемма 2. Если степенной ряд $q(z)=z+O\left(z^{2}\right)$ имеет челочисленные коэффициенты, то его обращение $z(q)=q+O\left(q^{2}\right)$ обладает тем же свойством.

Из теорем 1,2 и леммы 2 вытекает целочисленность степенных разложений для большого класса зеркальных отображений. Так, полагая $N=8$ и $N=10$ в теореме 1 , мы 
получаем целочисленность зеркальньх отображений, отвечающих семействам гиперповерхностей

$$
\begin{aligned}
\mathscr{Q}_{\psi}^{(8)} & :=\left\{x_{1}^{2}+x_{2}^{8}+x_{3}^{8}+x_{4}^{8}+x_{5}^{8}-8 \psi x_{1} x_{2} x_{3} x_{4} x_{5}=0\right\} \subset \mathbb{P}^{4}[4,1,1,1,1] \\
\mathcal{Q}_{\psi}^{(10)} & :=\left\{x_{1}^{2}+x_{2}^{5}+x_{3}^{10}+x_{4}^{10}+x_{5}^{10}-10 \psi x_{1} x_{2} x_{3} x_{4} x_{5}=0\right\} \subset \mathbb{P}^{4}[5,2,1,1,1]
\end{aligned}
$$

соответственно в весовых проективных пространствах (подробную информацию об этих семействах можно найти, например, в [2, §4]). Отметим также, что для малых значений $N$ в теореме 1 или $N_{1} N_{2} \cdots N_{r}$ в теореме 2 (а именно, когда соответствующее дифференциальное уравнение имеет порядок 2 или 3 ) целочисленность ряда $z(q)$ объясняется тем, что представляемая рядом функция является модулярной как функция от $\tau=\frac{1}{\pi i} \log q($ см. $[8, \S 1])$.

Настоящая работа устроена следующим образом. В следующем пункте мы редуцируем доказательство теорем 1,2 к арифметической проблеме $p$-адического анализа. В п. 3 мы приводим доказательство лемм 1, 2 и необходимые для дальнейшего сведения из $p$-адической техники Дворка [5]. Наконец, в п. 4 мы доказьваем основные результаты этой работы.

Тематика настоящей статьи легла в основу серии специальных семинаров по диофантовым приближениям и трансцендентным числам, организованных на механико-математическом факультете МГУ. Автор благодарит всех участников этих семинаров за ряд ценных замечаний и упрошений, появившихся в результате подробного обсуждения доказательства теорем 1,2 .

2. $p$-адическая редукция теорем 1,2 . Пусть $p$ - простое число. Обозначим через $\operatorname{ord}_{p} \xi p$-адический порядок числа $\xi \in \mathbb{Q}$ (степень вхождения $p$ в несократимую дробь для $\xi$ ); величина $\operatorname{ord}_{p} \xi$ может принимать любое целочисленное значение, для $\xi=0$ полагаем $\operatorname{ord}_{p} \xi=+\infty$. Замыкание поля $\mathbb{Q}$ относительно неархимедовой нормы $|\xi|_{p}=p^{-\operatorname{ord}_{p} \xi}$ обозначается через $\mathbb{Q}_{p} ;$ все элементы $\zeta$ поля $\mathbb{Q}_{p}$ (и, в частности, все элементы исходного поля $\mathbb{Q}$ ) обладают однозначным разложением

$$
\zeta=p^{r}\left(c_{0}+c_{1} p+c_{2} p^{2}+\cdots+c_{n} p^{n}+\cdots\right),
$$

где $r=\operatorname{ord}_{p} \zeta \in \mathbb{Z}$ и $0 \leqslant c_{n}<p$ для всех $n=0,1,2, \ldots$ и сходимость в (13) понимается в смысле нормы $|\cdot|_{p}$ (см., например, [9, гл. I, §4]). Разложение (13) назьвается $p$-адической записью числа $\zeta$. Множество элементов $\zeta \in \mathbb{Q}_{p}$, удовлетворяющих условию $\operatorname{ord}_{p} \zeta \geqslant 0$, образует кольцо $\mathbb{Z}_{p}$. Класс чисел $\zeta \in \mathbb{Q}_{p}$, для которых $r \geqslant s$ в записи (13), обозначим через $O\left(p^{s}\right)$.

ЛЕмма 3. Пусть рациональное число $\xi$ является әлементом $\mathbb{Z}_{p}$ для любого простого р. Тогда $\xi-$ иелое число.

ДокАЗАТЕльство. Достаточно привести доказательство для $\xi \neq 0$. Разложения числителя и знаменателя несократимой дроби для $\xi$ на простые множители индуцируют разложение на простые самого числа: $\xi=p_{1}^{s_{1}} p_{2}^{s_{2}} \cdots p_{l}^{s_{l}}$. При этом $\operatorname{ord}_{p_{j}} \xi=s_{j} \geqslant 0$ для $j=1, \ldots, l$, поскольку $\xi \in \mathbb{Z}_{p_{j}}$ по условию. Тем самым, число $\xi$ является произведением простых чисел в целых неотрицательных степенях, т.е. $\xi \in \mathbb{Z}$, что и требовалось. 
СлЕДСтвиЕ. Для доказательства теорем 1, 2 достаточно показать, что

$$
q(z)=z \cdot \exp \left(\frac{g(z)}{f(z)}\right) \in \mathbb{Z}_{p}[[z]]
$$

для любого простого $p$. (Здесь $\mathbb{Z}_{p}[[z]]$ обозначает кольцо формальных степенных рядов относительно z с коэффичиентами из $\mathbb{Z}_{p}$.)

3. Предварительные сведения. Прежде всего, докажем вспомогательные утверждения из п. 1.

ДокАЗАТЕЛЬСТво ЛЕммы 2. Для заданных степенньх рядов

$$
q(z)=\sum_{m=1}^{\infty} a_{m} z^{m}, \quad a_{1}=1, \quad a_{m} \in \mathbb{Z}, m=1,2, \ldots, \quad z(q)=\sum_{n=1}^{\infty} b_{n} q^{n}
$$

по условию вьполнено тождество $q=q(z(q))$, откуда

$$
q=\sum_{n=1}^{\infty} b_{n} q^{n}+a_{2}\left(\sum_{n=1}^{\infty} b_{n} q^{n}\right)^{2}+a_{3}\left(\sum_{n=1}^{\infty} b_{n} q^{n}\right)^{3}+\cdots .
$$

Сравнивая первые коэффициенты при степенях $q$ в (14), получаем $b_{1}=1, b_{2}+a_{2}=0$, $b_{3}+2 a_{2} b_{2}+a_{3}=0$, т.е. $b_{1}, b_{2}, b_{3}$ - целые числа. Дальнейшее доказательство проводим по индукции. Предположим теперь, что целочисленность $b_{1}, \ldots, b_{n-1}$ для $n \geqslant 3$ уже доказана, и рассмотрим коэффищиент при $q^{n}$ в (14). Он равен

$$
b_{n}+\sum_{i=2}^{n-1} a_{i} M_{i}+a_{n}=0
$$

где $M_{i}-$ коэффициент при $q^{n}$ многочлена $\left(b_{1} q+b_{2} q^{2}+\cdots+b_{n-1} q^{n-1}\right)^{i}, i=2, \ldots, n-1$. Согласно индукционному предположению числа $M_{i}$ являются цельми, так что соотношение (15) влечет целочисленность $b_{n}$. Лемма доказана.

ДокаЗАТЕЛЬСтво ЛЕммы 1. Пусть целое число $q, 0<q<N$, взаимно просто с $N$. Тогда в разложении на простые знаменатели чисел

$$
N^{m} \cdot \frac{(q / N)_{m}}{m !}=\frac{q(q+N)(q+2 N) \cdots(q+(m-1) N)}{m !}, \quad m=0,1,2, \ldots,
$$

содержат только простые делители числа $N$ (см., например, [10, гл. I, приложение]). Числители чисел (16) взаимно просты с $N$, а степень вхождения простого $p \mid N$ в $m$ ! равна

$$
\operatorname{ord}_{p} m !=\left[\frac{m}{p}\right]+\left[\frac{m}{p^{2}}\right]+\left[\frac{m}{p^{3}}\right]+\cdots<\frac{m}{p-1}
$$

(здесь $[\cdot]$ - целая часть числа). Поэтому постоянная (2) действительно сокращает знаменатели чисел

$$
\frac{\left(q_{1} / N\right)_{m}\left(q_{2} / N\right)_{m} \cdots\left(q_{k} / N\right)_{m}}{m !^{k}} .
$$

Лемма доказана. 
ЛЕмма 4. Для әлементов последовательности (3) справедливо следующее “факториальное" представление:

$$
A(m)=\frac{\left(a_{1} m\right) !\left(a_{2} m\right) ! \cdots\left(a_{\mu} m\right) !}{\left(b_{1} m\right) !\left(b_{2} m\right) ! \cdots\left(b_{\eta} m\right) !}, \quad m=0,1,2, \ldots,
$$

əде

$$
\begin{gathered}
\left\{a_{j}\right\}_{j=1, \ldots, \mu}=\left\{N, \frac{N}{p_{j_{1}} p_{j_{2}}}, \frac{N}{p_{j_{1}} p_{j_{2}} p_{j_{3}} p_{j_{4}}}, \ldots\right\}_{1 \leqslant j_{1}<j_{2}<\cdots \leqslant l}, \\
\left\{b_{i}\right\}_{i=1, \ldots, \eta}=\left\{1, \ldots, 1, \frac{N}{p_{j_{1}}}, \frac{N}{p_{j_{1}} p_{j_{2}} p_{j_{3}}}, \ldots\right\}_{1 \leqslant j_{1}<j_{2}<\cdots \leqslant l}
\end{gathered}
$$

- иелочисленные наборы, отвечающие данному $N=p_{1}^{s_{1}} p_{2}^{s_{2}} \cdots p_{l}^{s_{l}}$, $и$, кроме того,

$$
a_{1}+a_{2}+\cdots+a_{\mu}=b_{1}+b_{2}+\cdots+b_{\eta}
$$

ДокАЗАТЕЛЬСТво. Используя модификацию принципа формальной логики (см. [1, отд. 8 , задачи 21-25]), для целого $n \geqslant 0$ получаем

$$
\begin{aligned}
& \prod_{\substack{1 \leqslant q \leqslant N \\
(q, N)=1}}\left(\frac{q}{N}+n\right)=\prod_{1 \leqslant q \leqslant N}\left(\frac{q}{N}+n\right) \cdot\left(\prod_{\substack{1 \leqslant j \leqslant l \\
1 \leqslant q \leqslant N}} \prod_{\substack{1 \leqslant j \\
p_{j} \mid q}}\left(\frac{q}{N}+n\right)\right)^{-1} \\
& \times\left(\prod_{1 \leqslant j_{1}<j_{2} \leqslant l} \prod_{\substack{1 \leqslant q \leqslant N \\
p_{j_{1}} p_{j_{2}} \mid q}}\left(\frac{q}{N}+n\right)\right) \\
& \times\left(\prod_{1 \leqslant j_{1}<j_{2}<j_{3} \leqslant l} \prod_{\substack{1 \leqslant q \leqslant N \\
p_{j_{1}} p_{j_{2}} p_{j_{3}} \mid q}}\left(\frac{q}{N}+n\right)\right)^{-1} \cdots \\
& =\prod_{1 \leqslant q \leqslant N}\left(\frac{q}{N}+n\right) \cdot\left(\prod_{1 \leqslant j \leqslant l} \prod_{1 \leqslant q \leqslant N / p_{j}}\left(\frac{q}{N / p_{j}}+n\right)\right)^{-1} \\
& \times\left(\prod_{1 \leqslant j_{1}<j_{2} \leqslant l \leqslant q \leqslant N /\left(p_{j_{1}} p_{j_{2}}\right)}\left(\frac{q}{N /\left(p_{j_{1}} p_{j_{2}}\right)}+n\right)\right) \\
& \times\left(\prod_{1 \leqslant j_{1}<j_{2}<j_{3} \leqslant l \leqslant q \leqslant N /\left(p_{j_{1}} p_{j_{2}} p_{j_{3}}\right)}\left(\frac{q}{N /\left(p_{j_{1}} p_{j_{2}} p_{j_{3}}\right)}+n\right)\right)^{-1} \cdots .
\end{aligned}
$$

Домножим найденное выражение на

$$
\begin{gathered}
N^{-N} \cdot\left(\prod_{1 \leqslant j \leqslant l}\left(\frac{N}{p_{j}}\right)^{N / p_{j}}\right)^{-1} \cdot\left(\prod_{1 \leqslant j_{1}<j_{2} \leqslant l}\left(\frac{N}{p_{j_{1}} p_{j_{2}}}\right)^{N /\left(p_{j_{1}} p_{j_{2}}\right)}\right) \\
\quad \times\left(\prod_{1 \leqslant j_{1}<j_{2}<j_{3} \leqslant l}\left(\frac{N}{p_{j_{1}} p_{j_{2}} p_{j_{3}}}\right)^{N /\left(p_{j_{1}} p_{j_{2}} p_{j_{3}}\right)}\right)^{-1} \cdots=C_{N},
\end{gathered}
$$

возьмем произведение по всем $n=0,1, \ldots, m-1$ и разделим на $m !^{k}$. Тогда с учетом $(3)$ получим требуемое тождество (18). Лемма доказана.

До конца этого пункта мы фиксируем произвольное простое число $p$. 
ЛЕмма Дворка [9, гл. VI, §2, лемма 3], [11, гл. 14, 2$].$ Пусть $F(z) \in 1+z \mathbb{Q}[[z]]$. Тогда $F(z) \in 1+z \mathbb{Z}_{p}[[z]]$ в том и только том случае, когда

$$
\frac{F\left(z^{p}\right)}{F(z)^{p}} \in 1+p z \mathbb{Z}_{p}[[z]]
$$

Несмотря на то, что доказательство следуюшего утверждения содержится (с опечаткой) в [4], мы приводим его в данной работе для полноты изложения.

ЛЕмма $5\left[4\right.$, следствие 6.7]. Пусть $f(z) \in z \mathbb{Q}[[z]]$. Тогда $e^{f(z)} \in 1+z \mathbb{Z}_{p}[[z]]$ в том и только том случае, когда

$$
f\left(z^{p}\right)-p f(z) \in p z \mathbb{Z}_{p}[[z]] .
$$

ДоКАЗАТЕЛЬСТВо. Положим $F(z)=e^{f(z)} \in 1+z \mathbb{Q}[[z]]$.

Необходимость. Пусть $F(z) \in 1+z \mathbb{Z}_{p}[[z]]$. Тогда согласно лемме Дворка

$$
e^{f\left(z^{p}\right)-p f(z)}=\frac{F\left(z^{p}\right)}{F(z)^{p}}=1-p G(z)
$$

для некоторого степенного ряда $G(z) \in z \mathbb{Z}_{p}[[z]]$. Следовательно,

$$
f\left(z^{p}\right)-p f(z)=\log (1-p G(z))=-\sum_{m=1}^{\infty} \frac{p^{m} G(z)^{m}}{m} \in p z \mathbb{Z}_{p}[[z]],
$$

где мы воспользовались тем, что $p^{m} / m \in p \mathbb{Z}_{p}$ для всех целых $m \geqslant 1$.

Достаточность. Пусть теперь $f\left(z^{p}\right)-p f(z)=p H(z)$ для некоторого $H(z) \in z \mathbb{Z}_{p}[[z]]$. Поскольку для целого $m \geqslant 1$ степень вхождения простого $p$ в $m$ ! всегда меньие $m$ (см. (17)), мы вправе заключить, что

$$
\frac{F\left(z^{p}\right)}{F(z)^{p}}=e^{p H(z)}=1+\sum_{m=1}^{\infty} \frac{p^{m}}{m !} H(z)^{m} \in 1+p z \mathbb{Z}_{p}[[z]] .
$$

Применение леммы Дворка дает требуемое включение $e^{f(z)}=F(z) \in 1+z \mathbb{Z}_{p}[[z]]$. Лемма доказана.

ЛЕмма 6. Пусть коэффициенты степенного ряда

$$
f(z)=\sum_{m=0}^{\infty} A(m) z^{m} \in 1+z \mathbb{Z}_{p}[[z]], \quad A(m) \neq 0, \quad m=1,2, \ldots,
$$

для любых челых неотрицательных $u, v, n, s$ таких, что $0 \leqslant u<p^{s} u 0 \leqslant v<p$, удовлетворяют условию

$$
\frac{A\left(v+u p+n p^{s+1}\right)}{A(v+u p)}-\frac{A\left(u+n p^{s}\right)}{A(u)} \in p^{s+1} \mathbb{Z}_{p}
$$

Положимм

$$
f_{\nu}(z)=\sum_{m=\nu}^{\infty} A(m) z^{m}, \quad \nu=1,2, \ldots .
$$

Тогда для любого челого положстельного р справедливо сравнение

$$
\frac{f_{\nu}\left(z^{p}\right)}{f\left(z^{p}\right)} \equiv \frac{f_{\nu p}(z)}{f(z)}\left(\bmod \nu p \mathbb{Z}_{p}[[z]]\right) .
$$


ДокАЗАТЕльСтво. Выберем произвольное целое положительное $\nu$ и положим $s=$ $\operatorname{ord}_{p} \nu$. Тогда $\nu=n p^{s}$ для некоторого целого положительного $n$ и $\nu p \mathbb{Z}_{p}[[z]]=p^{s+1} \mathbb{Z}_{p}[[z]]$.

Полагая в теореме 1.1 из [5]

$$
A^{(r)}(m)=A(m), \quad g_{r}(m)=1, \quad r=0,1,2, \ldots, \quad m=0,1,2, \ldots,
$$

согласно условию (21) получаем сравнение

$$
f(z) \sum_{m=k p^{s}}^{(k+1) p^{s}-1} A(m) z^{m p} \equiv f\left(z^{p}\right) \sum_{m=k p^{s+1}}^{(k+1) p^{s+1}-1} A(m) z^{m}\left(\bmod p^{s+1} \mathbb{Z}_{p}[[z]]\right),
$$

справедливое при всех целых положительных $k$. Суммируя сравнения (24) по всем $k=n, n+1, n+2, \ldots$, заключаем, что

$$
f(z) f_{\nu}\left(z^{p}\right) \equiv f\left(z^{p}\right) f_{\nu p}(z)\left(\bmod p^{s+1} \mathbb{Z}_{p}[[z]]\right) .
$$

Наконец, поскольку $f(z) \in 1+z \mathbb{Z}_{p}[[z]]$, а значит и $f\left(z^{p}\right) \in 1+z \mathbb{Z}_{p}[[z]]$, мы можем умножить обе части сравнения $(25)$ на степенной ряд $\left(f(z) f\left(z^{p}\right)\right)^{-1} \in 1+z \mathbb{Z}_{p}[[z]]$, и это в точности отвечает требуемому сравнению (23). Лемма доказана.

ПРЕДЛОЖЕНИЕ 1. Пусть коэффициенты степенного ряда (20) для любых иелых неотричательных $u, v, n, s$ таких, что $0 \leqslant u<p^{s} u 0 \leqslant v<p$, удовлетворяют условию (21), а ряд $g(z)$ определяется разложением

$$
g(z)=\sum_{m=1}^{\infty} A(m) D(1, m) z^{m}=\sum_{m=1}^{\infty} A(m)\left(\sum_{\nu=1}^{m} \frac{1}{\nu}\right) z^{m} .
$$

Tогда $e^{g(z) / f(z)} \in \mathbb{Z}_{p}[[z]]$.

ДокАЗАТЕЛЬСТво. Меняя суммирование в (26), приходим к разложению

$$
g(z)=\sum_{\nu=1}^{\infty} \frac{1}{\nu} f_{\nu}(z)
$$

где ряды $f_{\nu}(z)$ определяются формулами (22). Следовательно,

$$
\begin{aligned}
\frac{g\left(z^{p}\right)}{f\left(z^{p}\right)}-p \frac{g(z)}{f(z)} & =\sum_{\nu=1}^{\infty} \frac{1}{\nu} \frac{f_{\nu}\left(z^{p}\right)}{f\left(z^{p}\right)}-p \sum_{\nu=1}^{\infty} \frac{1}{\nu} \frac{f_{\nu}(z)}{f(z)} \\
& =\sum_{\nu=1}^{\infty} \frac{1}{\nu}\left(\frac{f_{\nu}\left(z^{p}\right)}{f\left(z^{p}\right)}-\frac{f_{\nu p}(z)}{f(z)}\right)-p \sum_{\substack{\nu=1 \\
p \nmid \nu}}^{\infty} \frac{1}{\nu} \frac{f_{\nu}(z)}{f(z)} \in p z \mathbb{Z}_{p}[[z]]
\end{aligned}
$$

где каждое слагаемое первой суммы в (27) лежит в $p \mathbb{Z}_{p}[[z]]$ по лемме 6 , а каждое слагаемое второй - в $\mathbb{Z}_{p}[[z]]$, так как $f_{\nu}(z) \in z \mathbb{Z}_{p}[[z]], f(z) \in 1+z \mathbb{Z}_{p}[[z]]$ и $1 / \nu \in \mathbb{Z}_{p}$ для любого целого $\nu$, взаимно простого с $p$. Полученное включение $(27)$ согласно лемме 5 означает, что $e^{g(z) / f(z)} \in \mathbb{Z}_{p}[[z]]$. Предложение доказано.

В дальнейшем нам понадобятся некоторые свойства $p$-адической гамма-функиии

$$
\Gamma_{p}(n)=(-1)^{n} \gamma_{p}(n), \quad \text { где } \gamma_{p}(n)=\prod_{\substack{k=1 \\ k, p)=1}}^{n-1} k
$$


Лемма 7. Для любого иелого $n \geqslant 0$ имеет место тождество

$$
\frac{(n p) !}{n !}=p^{n} \gamma_{p}(1+n p)
$$

ДоКАЗАТЕЛЬСТВо. Из определения (28) получаем

$$
\gamma_{p}(1+n p)=\frac{(n p) !}{p \cdot 2 p \cdot 3 p \cdots n p}=\frac{(n p) !}{n ! p^{n}}
$$

откуда следует требуемое тождество.

ЛЕмма 8 [11, лемма 1.1]. Для всех целых положительных $k, n, s$ выполнено

$$
\Gamma_{p}\left(k+n p^{s}\right) \equiv \Gamma_{p}(k)\left(\bmod p^{s}\right)
$$

Лемма 9. Пусть последовательность иельх $A(m)=A_{N}(m), m=0,1,2, \ldots$, определяется формулой (3). Тогда для любого иелого неотрицательного $m$

$$
\frac{A(m p)}{A(m)}=1+O(p)
$$

u, в частности, $\operatorname{ord}_{p} A(m p)=\operatorname{ord}_{p} A(m)$.

ДоКАЗАТЕЛЬСТво. Согласно леммам 7,8 для любого целого положительного $a$ получаем

$$
\begin{aligned}
\frac{(a m p) !}{(a m) !} & =p^{a m} \gamma_{p}(1+a m p)=p^{a m}(-1)^{1+a m p} \Gamma_{p}(1+a m p) \\
& =p^{a m}(-1)^{1+a m p} \Gamma_{p}(1)(1+O(p))=p^{a m}(-1)^{a m p}(1+O(p))
\end{aligned}
$$

откуда, пользуясь леммой 4, находим требуемое:

$$
\frac{A(m p)}{A(m)}=p^{\left(a_{1}+\cdots+a_{\mu}-b_{1}-\cdots-b_{\eta}\right) m}(-1)^{\left(a_{1}+\cdots+a_{\mu}-b_{1}-\cdots-b_{\eta}\right) m p}(1+O(p))=1+O(p) .
$$

Лемма доказана.

4. Доказательство теорем 1, 2. Для простого $p$ определим на множестве

$$
\mathscr{C}_{p}:=\left\{\xi \in \mathbb{Q}: \xi>0, \operatorname{ord}_{p} \xi \geqslant 0\right\}
$$

отображение

$$
': \mathscr{C}_{p} \rightarrow \mathscr{C}_{p}, \quad \xi \mapsto \xi^{\prime},
$$

по следующему правилу: число $p \xi^{\prime}-\xi$ является минимальным представителем класса вычетов $-\xi(\bmod p)$ (иньми словами, $\xi^{\prime}-$ минимальный элемент $\mathscr{C}_{p}$ такой, что $\left.p \xi^{\prime}-\xi \in \mathbb{Z}\right)$.

Пусть $N \geqslant 2$ целое; числа $q_{1}, q_{2}, \ldots, q_{k}$ образуют полный набор остатков при делении на $N$, взаимно простых с $N$. 
ЛЕмма 10. Если простое р не делит $N$, то отображсение (29) является биекиией множества $\left\{q_{j} / N\right\}_{j=1, \ldots, k}$ на себя.

ДокАзАТЕЛЬСтво. Обозначим через $q_{j}^{\prime} / N$ образ элемента $q_{j} / N, j=1, \ldots, k$, при отображении (29). Согласно определению выполнено

$$
p q_{j}^{\prime} \equiv q_{j}(\bmod N), \quad j=1, \ldots, k
$$

Поскольку $p$ взаимно просто с $N$, существует $p^{\prime}$ такое, что $p p^{\prime} \equiv 1(\bmod N)$. Следовательно,

$$
\left\{q_{j}^{\prime}\right\}_{j=1, \ldots, k} \equiv\left\{p^{\prime} q_{j}\right\}_{j=1, \ldots, k} \equiv\left\{q_{j}\right\}_{j=1, \ldots, k}(\bmod N),
$$

откуда ввиду минимальности $q_{j}^{\prime}>0$ получаем требуемое утверждение.

ПРЕДЛОЖЕНИЕ 2. Пусть простое $р$ не является делителем $N$ в условиях теоремы 1 или делителем $N_{1} N_{2} \cdots N_{r}$ в условиях теоремы 2. Тогда для степенного ряда $q(z)$ из формулировки соответствующей теоремы справедливо включение $q(z) \in \mathbb{Z}_{p}[[z]]$.

ДокАЗАТЕльство. Множество параметров $\left\{q_{j} / N\right\}_{j=1, \ldots, k}$ обобщенного гипергеометрического ряда

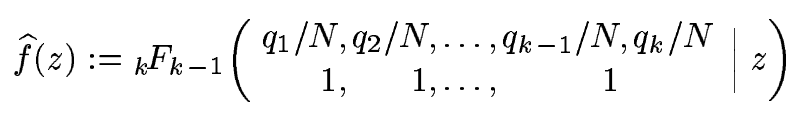

инвариантно относительно преобразования (29) по лемме 10. Полагая

$$
\widehat{g}(z):=\sum_{m=1}^{\infty} D_{N}(m) \frac{\left(q_{1} / N\right)_{m}\left(q_{2} / N\right)_{m} \cdots\left(q_{k} / N\right)_{m}}{m !^{k}} z^{m}
$$

согласно [5, теорема 4.1] получаем сравнение

$$
\frac{\widehat{g}\left(z^{p}\right)}{\widehat{f}\left(z^{p}\right)} \equiv p \frac{\widehat{g}(z)}{\widehat{f}(z)}\left(\bmod p \mathbb{Z}_{p}[[z]]\right)
$$

откуда, применяя лемму 5, заключаем, что $e^{\widehat{g}(z) / \widehat{f}(z)} \in \mathbb{Z}_{p}[[z]]$. Следовательно,

$$
q(z)=z \cdot \exp \left(\frac{g(z)}{f(z)}\right)=z \cdot \exp \left(\frac{\widehat{g}\left(C_{N} z\right)}{\widehat{f}\left(C_{N} z\right)}\right) \in z \mathbb{Z}_{p}\left[\left[C_{N} z\right]\right]=z \mathbb{Z}_{p}[[z]]
$$

в теореме 1 , где мы воспользовались взаимной простотой постоянной $C_{N}$ из $(2)$ и числа $p$.

Все рассуждения с необходимой модификацией степенных разложений $(30),(31)$ и заменой $C_{N}$ на $C_{N_{1}} C_{N_{2}} \cdots C_{N_{r}}$ остаются в силе и для ряда $q(z)$ в теореме 2 . Тем самым, требуемое предложение доказано полностью.

Таким образом, нам остается доказать редуцированные версии теорем 1,2 только для простых $p$, делящих $N$ или $N_{1} N_{2} \cdots N_{r}$ соответственно. 
ЛЕмма 11. Пусть $p$ - простой делитель числа $N$, последовательность иельх $A(m)=A_{N}(m), m=0,1,2, \ldots$, определяется формулой (3). Тогда для любых иелых неотрицательных $u, n, s, 0 \leqslant u<p^{s}$, выполнено

$$
\operatorname{ord}_{p} \frac{A\left(u+n p^{s}\right)}{A(u)} \geqslant 0 .
$$

ДокАЗАтЕльство. Поскольку $p \mid N$, для всех цельх неотрицательных $v, m, 0 \leqslant$ $v<p$, согласно $(3)$ имеем

$$
\begin{aligned}
\frac{A(v+m p)}{A(m p)} & =D_{N}^{v} \prod_{i=1}^{v} \frac{\left(q_{1}+(i-1) N+m N p\right) \cdots\left(q_{k}+(i-1) N+m N p\right)}{i+m p} \\
& =D_{N}^{v} \frac{\left(q_{1} \cdots q_{k}\right)^{v}}{v !}(1+O(p)),
\end{aligned}
$$

где

$$
D_{N}=N^{-k} \cdot C_{N}=\prod_{p \mid N} p^{k /(p-1)} .
$$

Следовательно,

$$
\operatorname{ord}_{p} \frac{A(v+m p)}{A(m p)}=\operatorname{ord}_{p} D_{N}^{v}=\frac{k v}{p-1}
$$

Воспользуемся для доказательства леммы методом математической индукции. При $s=0$ имеем $u=0$, так что оценка (32) принимает вид $\operatorname{ord}_{p} A(n) \geqslant 0$, а последнее неравенство следует из леммы 1.

Докажем теперь оценку (32) для $s \geqslant 1$, считая ее доказанной для меньших значений $s$. Представим число $u$ в виде $u=v+u_{1} p$, где $0 \leqslant v<p$ и $0 \leqslant u_{1}<p^{s-1}$. Тогда, применяя (34) и лемму 9, получаем

$$
\begin{aligned}
\operatorname{ord}_{p} \frac{A\left(u+n p^{s}\right)}{A(u)} & =\operatorname{ord}_{p} A\left(v+u_{1} p+n p^{s}\right)-\operatorname{ord}_{p} A\left(v+u_{1} p\right) \\
& =\left(\operatorname{ord}_{p} A\left(u_{1} p+n p^{s}\right)-\frac{k v}{p-1}\right)-\left(\operatorname{ord}_{p} A\left(u_{1} p\right)-\frac{k v}{p-1}\right) \\
& =\operatorname{ord}_{p} A\left(u_{1}+n p^{s-1}\right)-\operatorname{ord}_{p} A\left(u_{1}\right)=\operatorname{ord}_{p} \frac{A\left(u_{1}+n p^{s-1}\right)}{A\left(u_{1}\right)} \geqslant 0
\end{aligned}
$$

где последнее неравенство следует из индукционного предположения. Тем самым, оценка (32) доказана для любых целых неотрицательных $u, n, s, 0 \leqslant u<p^{s}$.

Лемма 12. Пусть простое $р$ делит $N$. Тогда әлементы последовательности (3) для любых иелых неотричательных $u, v, n, s$ таких, что $0 \leqslant u<p^{s} u$ $0 \leqslant v<p$, удовлетворяют условию (21). 
ДокАЗАТЕЛЬСТво. Имеем

$$
\begin{aligned}
& \frac{A\left(v+u p+n p^{s+1}\right)}{A\left(u p+n p^{s+1}\right)} \\
& \quad=\prod_{i=1}^{v} \frac{C_{N}\left(q_{1} / N+i-1+u p+n p^{s+1}\right) \cdots\left(q_{k} / N+i-1+u p+n p^{s+1}\right)}{i+u p+n p^{s+1}} \\
& =D_{N}^{v} \prod_{i=1}^{v} \frac{\left(q_{1}+(i-1) N+u N p+n N p^{s+1}\right) \cdots\left(q_{k}+(i-1) N+u N p+n N p^{s+1}\right)}{i+u p+n p^{s+1}} \\
& =D_{N}^{v} \prod_{i=1}^{v} \frac{\left(q_{1}+(i-1) N+u N p\right) \cdots\left(q_{k}+(i-1) N+u N p\right)}{i+u p}\left(1+O\left(p^{s+1}\right)\right) \\
& =\frac{A(v+u p)}{A(u p)}\left(1+O\left(p^{s+1}\right)\right)
\end{aligned}
$$

где постоянная $D_{N}$ определена в $(33)$.

Теперь мы воспользуемся факториальньм представлением (18) и свойствами $p$-адической гамма-функции. Согласно лемме 7 для любого целого положительного $a$ вьполнено

$$
\frac{(a u p) !}{(a u) !}=p^{a u} \gamma_{p}(1+a u p)
$$

и

$$
\begin{aligned}
\frac{\left(a\left(u p+n p^{s+1}\right)\right) !}{\left(a\left(u+n p^{s}\right)\right) !} & =p^{a\left(u+n p^{s}\right)} \gamma_{p}\left(1+a u p+a n p^{s+1}\right) \\
& =p^{a\left(u+n p^{s}\right)}(-1)^{1+a u p+a n p^{s+1}} \Gamma_{p}\left(1+a u p+a n p^{s+1}\right) \\
& =p^{a u+a n p^{s}}(-1)^{1+a u p+a n p^{s+1}} \Gamma_{p}(1+a u p)\left(1+O\left(p^{s+1}\right)\right) \\
& =(-p)^{a n p^{s}} \frac{(\text { aup }) !}{(a u) !}\left(1+O\left(p^{s+1}\right)\right)
\end{aligned}
$$

где мы также применили лемму 8. Пользуясь теперь леммой 4 и соотношениями (36) для $a \in\left\{a_{1}, \ldots, a_{\mu}, b_{1}, \ldots, b_{\eta}\right\}$, находим

$$
\begin{aligned}
\frac{A\left(u p+n p^{s+1}\right)}{A\left(u+n p^{s}\right)} & =\frac{A(u p)}{A(u)}\left(1+O\left(p^{s+1}\right)\right) \prod_{j=1}^{\mu}(-p)^{a_{j} n p^{s}} \prod_{i=1}^{\mu}(-p)^{-b_{i} n p^{s}} \\
& =\frac{A(u p)}{A(u)}\left(1+O\left(p^{s+1}\right)\right)
\end{aligned}
$$

согласно (19).

После перемножения соотношений (35) и (37) получаем

$$
\frac{A\left(v+u p+n p^{s+1}\right)}{A\left(u+n p^{s}\right)}=\frac{A(v+u p)}{A(u)}\left(1+O\left(p^{s+1}\right)\right),
$$

откуда

$$
\frac{A\left(v+u p+n p^{s+1}\right)}{A(v+u p)}=\frac{A\left(u+n p^{s}\right)}{A(u)}\left(1+O\left(p^{s+1}\right)\right) .
$$

Согласно лемме 11 правая часть (38) лежит в $\mathbb{Z}_{p}$, откуда и следует включение (21). Лемма доказана. 
СлЕДСТВИЕ. Пусть простое $p$ делит каждое из чисел $N_{1}, N_{2}, \ldots, N_{r}$. Тогда әлементы последовательности (8) для любых иелых неотричательных $u, v, n, s$ таких, что $0 \leqslant u<p^{s} u 0 \leqslant v<p$, удовлетворяют условию (21).

ДокАЗАТЕЛЬСТво. Соотношения (38) и (32) справедливы для любой из последовательностей $A(m)=A_{N_{j}}(m), j=1, \ldots, r, m=0,1,2, \ldots$ Отсюда мы получаем включение (21) для элементов последовательности (8).

ПРЕДЛОЖЕНИЕ 3. Пусть простое $р$ является делителем $N$ в условиях теоремы 1 или делителем $N_{1} N_{2} \cdots N_{r}$ в условиях теоремы 2. Тогда для степенного ряда $q(z)$ из формулировки соответствующей теоремы справедливо включение $q(z) \in \mathbb{Z}_{p}[[z]]$.

ДоКАЗАТЕЛЬСТво. Для простоты мы ограничимся доказательством предложения при вьполнении условий теоремы 1. Схема рассуждений остается неизменной и в общем случае, когда простое $p$ делит каждое $N_{j}, j=1, \ldots, r$.

Представим функцию (6) в виде суммы $g(z)=g_{1}(z)+g_{2}(z)$, где

$$
g_{1}(z)=\sum_{m=1}^{\infty} A(m) \sum_{j=1}^{k} D\left(\frac{q_{j}}{N}, m\right) z^{m}, \quad g_{2}(z)=-k \sum_{m=1}^{\infty} A(m) D(1, m) z^{m}
$$

и докажем, что $e^{g_{i}(z) / f(z)} \in \mathbb{Z}_{p}[[z]], i=1,2$.

Отметим, что

$$
D\left(\frac{q_{j}}{N}, m\right)=N \sum_{n=1}^{m} \frac{1}{q_{j}+(n-1) N} \in p \mathbb{Z}_{p}, \quad j=1, \ldots, k, \quad m=1,2, \ldots
$$

поскольку $N$ делится на $p$, а знаменатель каждого слагаемого в (39) взаимно прост с $N$ (и, значит, с $p$ ). Кроме того, все элементы последовательности (3) лежат в $\mathbb{Z}_{p}$, $f(z) \in 1+z \mathbb{Z}_{p}[[z]]$. Следовательно, $g_{1}(z) \in p z \mathbb{Z}_{p}[[z]]$ и $g_{1}(z) / f(z) \in p z \mathbb{Z}_{p}[[z]]$, так что для ряда $g_{1}(z) / f(z)$ условия леммы 5 вьполнены. В силу леммы 5 мы получаем включение $e^{g_{1}(z) / f(z)} \in \mathbb{Z}_{p}[[z]]$.

Согласно лемме 12 элементы последовательности (3) после домножения на целое число $-k$ удовлетворяют условию (21). Поэтому из предложения 1 следует включение $e^{g_{2}(z) / f(z)} \in \mathbb{Z}_{p}[[z]]$.

Окончательно,

$$
q(z)=z e^{g(z) / f(z)}=z e^{g_{1}(z) / f(z)} \cdot z e^{g_{2}(z) / f(z)} \in z \mathbb{Z}_{p}[[z]]
$$

что и требовалось.

Согласно следствию из леммы 3 применение предложений 2,3 доказьвает теоремы 1 и 2. 


\section{СПИСОК ЦИТИРОВАННОЙ ЛИТЕРАТУРЫ}

[1] Полиа Г., Сеге Г. Задачи и теоремы из анализа. Ч. II. 3-е изд. М.: Наука, 1978.

[2] Morrison D.R. Picard-Fuchs equations and mirror maps for hypersurfaces // Essays on Mirror Manifolds / ed. S.-T. Yau. Hong Kong: International Press, 1992. P. 241-264; _ // Mirror Symmetry I / ed. S.-T. Yau. AMS/IP Stud. Adv. Math. V. 9. Providence, R.I.: Amer. Math. Soc., 1998. P. 185-199.

[3] Batyrev V. V., van Straten D. Generalized hypergeometric functions and rational curves on Calabi-Yau complete intersections in toric varieties // Comm. Math. Phys. 1995. V. 168. № 3. P. 493-533.

[4] Lian B.H., Yau S.-T. Mirror maps, modular relations and hypergeometric series I // E-print http://arXiv.org/abs/hep-th/9507151; Integrality of certain exponential series // Lectures in Algebra and Geometry / ed. M.-C. Kang. Proceedings of the International Conference on Algebra and Geometry, National Taiwan University (Taipei, Taiwan, December 26-30, 1995). Cambridge, MA: International Press, 1998. P. 215-227.

[5] Dwork B. On $p$-adic differential equations IV. Generalized hypergeometric functions as $p$-adic analytic functions in one variable // Ann. Sci. École Norm. Sup. (4). 1973. V. 6. № 3. P. 295-315.

[6] Katz N. M. Algebraic solutions of differential equations ( $p$-curvature and the Hodge fibration) // Invent. Math. 1972. V. 18. №1/2. P. 1-118.

[7] Morrison D. R. Mathematical aspects of mirror symmetry // Complex Algebraic Geometry / ed. J. Kollár. Lectures of a Summer Program (Park City, UT, 1993), IAS/Park City Math. Ser. V. 3. Providence, RI: Amer. Math. Soc., 1997. P. 267-340.

[8] Zudilin W. Number theory casting a look at the mirror // Preprint, 2000 (submitted for publication); E-print http://arXiv.org/abs/math/0008237.

[9] Коблиц Н. p-адические числа, $p$-адический анализ и дзета-функции. М.: Мир, 1982.

[10] André Y. G-Functions and Geometry. Aspects Math. (A Publication of the Max-PlanckInstitut für Mathematik, Bonn). V. E13. Braunschweig: Vieweg, 1989.

[11] Lang S. Cyclotomic Fields. I, II (Combined 2nd edition). Graduate Texts in Math. V. 121. New York: Springer-Verlag, 1990.

Московский государственный университет им. М.В. Ломоносова

Поступило

E-mail : wadim@ips.ras.ru 